\title{
Study of Acute Effect of Caffeine on Cognition among Adults- An Exploratory Intervention Trial
}

\author{
Krishnan $\mathrm{S}^{1}$, Smisha Mohan ${ }^{2}$, Jeneth Berlin Raj $\mathrm{T}^{3}$, Manikandan $\mathrm{S}^{4}$ \\ ${ }^{1}$ Assistant Professor, Department of Physiology, All India Institute of Medical Sciences, Guwahati, ${ }^{2}$ PhD Scholar, \\ ${ }^{3}$ Professor \& Head, ${ }^{4}$ Tutor \& PhD Scholar, Department of Physiology, Mahatma Gandhi Medical College and \\ Research Institute, Sri Balaji Vidyapeeth, Pondicherry, India
}

\begin{abstract}
Introduction/Background: Information processing has substantial role in performing intellectual activities such as thinking, reasoning, remembering, imagining, or learning. Caffeine being a CNS stimulant improves mental performance, especially on alertness, attention, concentration and learning depending on the quantity of intake. In the present study, an attempt is made to study the effect of caffeine on cognitive processing in healthy individuals.
\end{abstract}

Methodology: This cross sectional study was conducted in 50 subjects at Mahatma Gandhi Medical College \& Research Institute. MOCA questionnaire was utilized to assess the level of cognition of each subject. Visual reaction time, auditory reaction time and critical fusion frequency prior and after consumption of 75 $\mathrm{mg}$ of caffeine in $200 \mathrm{ml}$ of milk.

Results: Both visual and auditory reaction time were significantly reduced $(\mathrm{p}<0.001)$ after intake of caffeine intake. The ability of the subject to appreciate the flickering light stimuli to be steady (critical flicker fusion frequency) was significantly improved $15 \%$ after caffeine intake.

Conclusion: Decrease in visual and auditory reaction and increase in critical flicker fusion frequency values indicate caffeine increases alertness thereby it may enhances performance efficiency in reasoning, planning, judgment, organizing, concept formation, and problem solving.

Key Words: Visual reaction time, Auditory reaction time, $M O C A$

\section{Introduction}

Cognition is the process of knowing, which includes awareness, perception, reasoning and judgment. Level of cognition can be assessed by reaction time. Reaction time (RT) explains about the fastness of the individual to any sort of stimulus. RT is defined as the interval of time

\section{Corresponding author details:}

Dr. S.Krishnan

Assistant Professor, Department of Physiology AIIMS, Guwahati, Mail:drkrish10@gmail.com Ph: +91 9791213830 between the presentation of the stimulus and appearance of appropriate voluntary response in the subject. It is a measure of function of sensorimotor association (1) and performance of an individual (2). It involves multidimensional tasks which includes processing, decision making, and response programming. The concept of RT was first described by AbūRayhān alBīrūnī(3). Dutch physiologist Franciscus Cornelis Donders was among the first to systematically measure human RT using a telegraph like device invented in 1840 by Charles Wheatstone. When the stimulus is 
applied, it activates the sensory neuron and reaches the brain or spinal cord. This will further activate the motor neuron to produce a response (4). RT can be affected by age, sex, left or right hand, central versus peripheral vision, practice, fatigue, fasting, breathing cycle, personality types, exercise, and intelligence of the subject $(5,6)$. Simple auditory reaction time is the time interval between the onset of the single stimulus and the initiation of the response under the condition that the subject has been instructed to respond as rapidly as possible (7). Caffeine is a central nervous system stimulant. It can temporarily make you feel more awake and energetic, but it can also give you the jitters. Caffeine reaches your brain quickly and acts as a central nervous system stimulant. The most noticeable effect is alertness. It may help the person to feel more awake and less tired, so it's a common ingredient in medications to treat drowsiness (8). Caffeine has shown to have cognitive-enhancing effects (9). Caffeine can help to improve mental performance, especially on alertness, attention and concentration depending on the level of intake (10). The effect of caffeine on cognition of the individual evaluated by RT is not well documented. Hence the present study will focus on effect of caffeine on cognition by using visual and auditory reaction time.

Aim: The aim of the study is to find the acute effect of caffeine on cognition

\section{Objective}

1. To estimate the acute effect of caffeine on visual reaction time

2. To estimate the acute effect of caffeine on auditory reaction time

3. To estimate the acute effect of caffeine on critical fusion frequency

4. To correlate the MoCA score with cognitive function

\section{Methodology}

This was a cross sectional study done on 50 subjects conducted in Mahatma Gandhi Medical College \&
Research Institute. A total sample size of 44 was calculated to detect a medium effect using $G$ power software $(\mathrm{d}=0.50)$. The power of the test was fixed at $90 \%$ and alpha at 0.05 (11). Institutional review committee (IRB) and Institutional Ethical committee (IEC) clearance was obtained from the institution before the commencement of the study (faculty project/08/2019/08). Informed consent was obtained from the subject. The age group of the study participants was from 30 to 40 Years. After recruiting the subject participant information was collected. The subjects were instructed to come on fasting and were advised to avoid any physical activity including yoga on the day of sample collection. Questionnaire was administered to get the general information along with history of caffeine intake (coffee/Tea/chocolate) about the subject. Montreal cognitive assessment (MoCA) questionnaire was administered to the study participants to assess the cognitive scores. Visual reaction time, auditory reaction time and critical fusion frequency was measured by using standard instruments (12). Pre and post reaction time measurement was taken from the subject to address the difference in reaction time. The subject was provided with $200 \mathrm{ml}$ of coffee. The coffee was prepared by mixing $2 \mathrm{~g}$ of coffee powder with caffeine concentration of $75 \mathrm{mg}$ with $200 \mathrm{ml}$ of milk. Sugar free capsules which were used will minimise the effect of sugar on reaction time. The participants will be instructed to take a cup of coffee. After 30 minutes post measurement of visual, auditory reaction time and critical fusion frequency time will be recorded. Based on the results of the study the acute effect of caffeine on cognition will be evaluated. Paired $t$ test will be used to for analysing the data using SPSS version 16. Post measurement of reaction time was collected after half an hour of coffee intake. The reaction time was measured in milliseconds (ms) and critical fusion frequency was measured in $(\mathrm{Hz})$. Data is represented as mean $\pm \mathrm{SD}$. Paired ' $\mathrm{t}$ ' test was applied for comparing the variables. Statistical significance was taken at 5\% significance level. The data was analysed using SPSS version 16. 


\section{PC 1000 Hertzs Reaction Timer:}

PC 1000 Hertzs is a Simple Reaction time measuring in house build device, used for measuring the auditory and visual reaction time. It's a 1000 hertz square wave oscillator which has a soft key for start and stop function. It has two components $(\mathrm{A} \& \mathrm{~B})$ connected to each other. First component (A) has a start button which will be out of the view from the subjects and it is controlled by the examiner only. Second component (B) has a stop button which will be operated by the subjects. Also it has a small red LED light for visual stimulus and head phone (1000 hertz's tone) which receives auditory stimulus respectively. Red light is selected for the experiment as it persists for a long time on retina. Component $\mathrm{A}$ and component $\mathrm{B}$ is in turn connected to a personal computer which has audacity sound software installed in it. Audacity software records the reaction time in 0.001 sec accuracy in wave format. The data file can be stored as a *. wav file for future reference.

\section{Visual Reaction Time (VRT) Measurement:}

Examiner presses the START button in first component (A) which will be out of the view of the subject .Subject is instructed to press the STOP button in second component (B) as soon as he/she sees the red light in the instrument. Reaction time is recorded in audacity software.

\section{Auditory Reaction Time (ART) Measurement:}

Examiner presses the start button (A) Which will be out of the view of the subject and the subject is instructed to press the stop button (B) as soon as he/she hears the sound (1000 hertz's tone) through the headphone connected to it. Reaction time is recorded in audacity software. Minimum three trials are given for both VRT and ART measurement. Minimum time recorded is calculated as final VRT and ART in msec.

\section{Critical Flicker Fusion Frequency (CFFF) device}

It has a built in and Light emitting diode (Red color- $5 \mathrm{~mm}$ diameter) driver circuitry. Its capable of working in the range of 10- $80 \mathrm{Hzs}$. The type of wave form which is used to drive the LED is square wave. This square wave output is provided by a license free software which is called as Audio Sweep Generator, (SweepGen.exe, V3.5.4.29, 2011 Mar 04) Through the Audio output port it drives the LED. The frequency can be changed from $10 \mathrm{~Hz}$ to $80 \mathrm{~Hz}$ in steps of $1 \mathrm{~Hz}$. The Frequency at which the subject experiences no flicker is noted to be the Critical flicker fusion frequency of the subject.

\section{Results}

The age group of our study participants was in the range of 30-40 years with mean height ranging $162 \pm 20.1$ $\mathrm{cms}$ and mean weight ranging $68.3 \pm 14.2 \mathrm{kgs}$. Among the study participants 20 were male and 29 were female. Visual Reaction time after consumption of coffee is significantly reduced when compared to reaction time before coffee intake $(p<0.001)($ Table 1$)$. There was $17 \%$ reduction in visual reaction time after the consumption of the coffee. Auditory Reaction time after consumption of coffee is significantly reduced when compared to reaction time before coffee intake $(\mathrm{p}<0.001)$ (Table 1). There was $19 \%$ reduction in auditory reaction time after the consumption of the coffee. Critical Fusion frequency after consumption of coffee is significantly increased when compared to before coffee intake $(p<0.001)$ (Table 1). There was $15 \%$ increase in critical fusion frequency after the consumption of the coffee.

There was a significant negative correlation between MOCA score and visual reaction time before and after consumption of coffee $(r=-0.616, p<0.001$ $\& \mathrm{r}-0.505,0.001)$ (Table 2). There was a significant negative correlation between MOCA score and auditory reaction time before and after consumption of coffee ( $\mathrm{r}=$ $-0.384, \mathrm{p}<0.01 \& \mathrm{r}-0.443, \mathrm{p}<0.003$ ) (Table 2). There was no significant correlation between MOCA and critical fusion frequency. 
Table 1: Comparison of visual and auditory reaction time before and after coffee intake

\begin{tabular}{|c|c|c|c|c|}
\hline S.NO & Parameter & Pre & Post & P Value \\
\hline 1 & VRT (ms) & $0.294 \pm 0.144$ & $0.221 \pm 0.134$ & 0.001 \\
\hline 3 & ART (ms) & $0.212 \pm 0.937$ & $0.171 \pm 0.579$ & 0.001 \\
\hline 5 & CFF(Hz) & $36.71 \pm 5.03$ & $42.51 \pm 6.006$ & 0.001 \\
\hline
\end{tabular}

Data represented as mean $\pm \mathrm{SD}$. $\mathrm{P}<0.05$ was considered as statistically significant. VRT-Visual Reaction Time. ART- Auditory Reaction Time. CFF- critical Fusion Frequency

Table 2: Correlation between MOCA and cognition

\begin{tabular}{|c|c|c|c|c|c|c|c|}
\hline \multicolumn{2}{|c|}{ S. No } & VRT - Pre & VRT - Post & ART - Pre & ART - Post & CFF- Pre & CFF- Post \\
\hline \multirow{2}{*}{ MOCA } & r value & -0.616 & -0.505 & -0.384 & -0.443 & 0.238 & 0.214 \\
\cline { 2 - 7 } & P value & 0.001 & 0.001 & 0.01 & 0.003 & 0.120 & 0.124 \\
\hline
\end{tabular}

. Pearson correlation was done. $\mathrm{P}<0.05$ was considered as statistically significant. VRT-Visual Reaction Time. ART- Auditory Reaction Time. CFF- Critical Fusion Frequency. MOCA- Montreal Cognitive Assessment

\section{Discussion}

This study was done in 50 subjects showed significant changes in the reaction time after coffee intake. The study helped us to understand the acute effect of caffeine on cognition. Caffeine being a central stimulant will help in enhancing the activity of central nervous system (13). There was a significant reduction in reaction time (visual \& auditory) and critical fusion frequency after coffee intake which explains that caffeine activates the central nervous system. This explains that caffeine intake was associated with increase in alertness. Our study results prove that caffeine in smaller doses can be beneficial to the humans by increasing the activity of CNS which in turn increases the alertness of the individual. There were only few studies done to evaluate the acute effect of caffeine on cognition and there were no published data in south India. There were conflicting results from the previous studies like some studies explaining that caffeine increases the alertness and improves the cognition and some studies are explaining vice versa (14). Our study results explained that the caffeine had significant positive effect on cognition. There was a significant correlation between MOCA score and post reaction time measurements in our study which explains that caffeine intake is strongly related to decrease in reaction time and increase the alertness. Caffeine in general causes stimulation of central nervous system which enhances cognition. The effect of caffeine on CNS varies depending on the dose of caffeine but it generally improves reaction time, wakefulness, concentration, and motor coordination (15). The amount of caffeine needed to produce these effects varies from person to person, depending on body size and degree of tolerance. (16) The effects will arise around one hour after consumption, and it will lasts for about three or four hours. Small doses of coffee can be given to shift workers which can reduce the effect of sleep deprivation by increasing the alertness. This can enhance the quality of the work and reduces the mortality (17). Apart from these there are some medical benefits of caffeine such 
as treatment of bronchopulmonary dysplasia, apnoea of prematurity (18). Small doses of caffeine can be consumed by the humans during the needful times like during exams, during continuous heavy work by the workers etc. Caffeine can improve the athletic performance and endurance in sports persons (19). Further studies with larger samples are needed to evaluate the chronic effects of caffeine on central nervous system and other health effects on humans which can explain the benefits to humans. If the effects of regular coffee are to be fully understood, it is important that future research compares these to the equivalent dose of caffeine, decaffeinated coffee, and placebo. Furthermore, research in this area must include plasma levels of potentially important compounds, including phenolic compounds. This would allow assessment of the impact of caffeine on the pharmacodynamic profile of other components in coffee. Further research is also required in which cognition is measured alongside potential underlying mechanisms including, but not limited to, glucoregulation and modulation of cerebral hemodynamics.

\section{Conclusion}

Caffeine a central nervous stimulant decreases the reaction time and increases the cognition. Small doses of caffeine can be consumed by the humans during the needful times like during exams, during continuous heavy work by the workers.

\section{Conflict of Interest -Nil}

Funding - Nil

\section{References}

1. Shenvi D, Balasubramanian P. A comparative study of visual and auditory reaction times in males and females. Indian J PhysiolPharmacol. 1994;38:22931

2. Das S, Gandhi A, Mondal S. Effect of premenstrual stress on audiovisual reaction time and audiogram. Indian $J$ PhysiolPharmacol. 1997;41:67-70.

3. Welford AT. Choice reaction time: Basic concepts. In: Welford AT, editor. Reaction Times. New York: Academic Press; 1980. pp. 73-128.
4. Duke-Elder S. FranciscusCornelisDonders. $\mathrm{Br} J$ Ophthalmol. 1959;43:65-8

5. Karia RM, Ghuntla TP, Mehta HB, Gokhale PA, Shah CJ. Effect of gender difference on visual reaction time: A study on medical students of Bhavnagar region. IOSR-PHR. 2012; 2:452-4.

6. Marieb Elaine N. Human Anatomy and Physiology Laboratory Manual (Cat Version) 7th ed. San Francisco, California: Benjamin Cummings; 2003. Exercise 22 Human reflex physiology. Activity 9: testing reaction time for basic and acquired reflexes; pp. 232-3

7. Brunyé T.T, Mahoney CR, Lieberman HR, Giles GE, Taylor HA. Acute caffeine consumption enhances the executive control of visual attention in habitual consumers. Brain Cogn, 2010; 74:18692.

8. Badwe N, Patil KB, Yelam SB, Vikhe BB, Vatve MS. A comparative study of hand reaction time to visual stimuli in students of 1 st MBBS of a rural medical college. Pravara Med Rev. 2012;4:4-6

9. Daniel Borota, Elizabeth Murray, GizemKeceli, Allen Chang, Joseph M Watabe, Maria Ly, John P Toscano, Michael A Yassa. Post-study caffeine administration enhances memory consolidation in humans.Nature Neuroscience.2014; 17: 201-203

10. SmitH.R, Rogers P.J.Effects of low doses of caffeine on cognitive performance, mood and thirst in low and higher caffeine consumers. Psychophymacology, 2000; 152:167-173.

11. Faul F, Erdfelder E, Lang AG, Buchner A. G*Power 3: A flexible statistical power analysis program for the social, behavioral, and biomedical sciences. Behav Res Methods. 2007;39:175-91.

12. Niruba, K.N. Maruthy. Assessment of Auditory and Visual Reaction Time in Type 2 Diabetics -A Case Control Study. Al Ameen J Med S ci. 2 011; 4 (3): $274-279$.

13. Nehlig A, Daval JL, Debry G (1992). "Caffeine and the central nervous system: mechanisms of action, biochemical, metabolic and psychostimulant effects". Brain Research. Brain Research Reviews. 17 (2): 139-70.

14. Crystal F. Haskell . David O. Kennedy Keith A. Wesnes . Andrew B. Scholey. Cognitive and mood improvements of caffeine in habitual consumers and habitual non-consumers of caffeine. 
Psychopharmacology. 2005.179: 813-825

15. Poleszak E, Szopa A, Wyska E, Kukuła-Koch W, Serefko A, Wośko S, Bogatko K, Wróbel A, Wlaź P .'Caffeine augments the antidepressant-like activity of mianserin and agomelatine in forced swim and tail suspension tests in mice". Pharmacological Reports.2016. 68 (1): 56-61.

16. Bolton S, Null G. "Caffeine: Psychological Effects, Use and Abuse" (PDF). Orthomolecular Psychiatry. 1981. 10 (3): 202-211.
Medico-legal Update, October-December 2021, Vol.21, No. $4 \mathbf{5 5}$

17. Snel J, Lorist MM. Effects of caffeine on sleep and cognition. Progress in Brain Research. 2011.190: 105-17.

18. Kugelman A, Durand M. A comprehensive approach to the prevention of bronchopulmonary dysplasia . Pediatric Pulmonology. 2011.46 (12): 1153-65.

19. Pesta DH, Angadi SS, Burtscher M, Roberts CK. The effects of caffeine, nicotine, ethanol, and tetrahydrocannabinol on exercise performance. 2013. Nutrition \& Metabolism. 10 (1): 71. 